\section{Multiresolution Approximation Using Shifted Splines}

Frank Müller, Patrick Brigger, Klaus Illgner, and Michael Unser

\begin{abstract}
We consider the construction of least squares pyramids using shifted polynomial spline basis functions. We derive the pre and postfilters as a function of the degree $n$ and the shift parameter $\Delta$. We show that the underlying projection operator is entirely specified by two transfer functions acting on the even and odd signal samples, respectively. We introduce a measure of shift invariance and show that the most favorable configuration is obtained when the knots of the splines are centered with respect to the grid points (i.e., $\Delta=1 / 2$ when $n$ is odd and $\Delta=0$ when $n$ is even). The worst case corresponds to the standard multiresolution setting where the spline spaces are nested.
\end{abstract}

\section{INTRODUCTION}

Splines have many nice properties that have contributed to their recent popularity for constructing wavelet bases and multiresolution signal approximations. They have a simple analytical form (piecewise polynomial) that facilitates their manipulation [1], [2]. They have excellent approximation properties, mainly because the underlying $B$-spline basis functions are very regular [3], [4]. Splines are also optimal in the sense that they provide the signal interpolant with the least oscillating energy [5]. Finally, the spline framework allows for a progressive transition between the two most extreme signal representations: the piecewise constant model (spline of degree zero), which uses the most localized but least regular basis functions, and the bandlimited model, which corresponds to a spline of infinite degree [6].

Several constructions of spline pyramids have been proposed in the literature. One approach, which falls into the multiresolution framework of the wavelet transform, minimizes the continuous $L_{2}$ norm [7], [8]. The continuous formulation imposes a nestedness constraint that does not allow for the shifting of the splines spaces: The knots (or spline discontinuities) need to be positioned on the integers. There is also an alternative design that considers the minimization of the $l_{2}$ norm [9], [10]; in that case, the basis functions are usually chosen to be symmetric with respect to the origin.

The purpose of this correspondence is to extend the $l_{2}$ construction of spline pyramids by considering spline models that may be shifted with respect to the finer grid. In doing so, we lose the nestedness property, which is not essential in the discrete formulation, but we gain in flexibility. For instance, we can design centered pyramids with a quadtree-like topology, i.e., where a coarse-level node is positioned in the center of its finer level predecessors. The proposed scheme also lends itself to the optimization of the shift parameter. Here, we will pay special attention to the issue of shift-invariance and determine the best $\Delta$ accordingly.

Manuscript received January 27, 1997; revised March 19, 1998. The associate editor coordinating the review of this paper and approving it for publication was Dr. Truong Q. Nguyen.

F. Müller and K. Ilgner are with the Institut für Elektrische Nachrichtentechnik, Rheinisch-Westfälische Hochschule (RWTH), Aachen, Germany.

P. Brigger is with the Biomedical Engineering and Instrumentation Program, National Center for Research Resources, National Institutes of Health, Bethesda, MD 20892-5766 USA.

M. Unser is with the EPFL, DMT/IOA, Biomedical Imaging Group, $\mathrm{CH}$ 1015, Lausanne, Switzerland (e-mail: Michael.Unser@epfl.ch).

Publisher Item Identifier S 1053-587X(98)05951-0.

\section{Discrete Spline APPRoximation}

We consider the construction of a dyadic spline pyramid in a purely discrete framework. The basic operation, which is applied iteratively, is the approximation from a fine space $S_{1}$ onto a coarse space $S_{2}$ at twice the scale. For mathematical convenience, we set $S_{1}=l_{2}$ (the space of finite energy sequences) and consider the coarser subspace $S_{1} \subset l_{2}$ generated from the even integer translates of a generating sequence $h(k)$

$$
S_{2}=\left\{\tilde{s}(k)=\sum_{l \in Z} s_{2}(l) h(k-2 l) \mid s_{2} \in l_{2}\right\} .
$$

We also require that $\{h(k-2 l)\}_{l \in Z}$ forms a Riesz basis of $S_{2}$ so that every sequence $\tilde{s}(k) \in S_{2}$ has a unique characterization in terms of its pyramid coefficients $s_{2}(l)$. In most pyramid constructions, the generating kernel $h$ is symmetric, and the coarser level nodes (or grid points) are positioned at the even integers. Here, we will shift these nodes by $\Delta$ and select $h$ to be the impulse response of the corresponding spline interpolator. In other words, we will synthesize the signal $\tilde{s}(k)$ by fitting its coefficients $s_{2}(l)$ with a polynomial spline of degree $n$ and resampling at twice the rate with a shift $\Delta$ (cf., Fig. 1). Using the spline signal processing formalism developed in [2], it is not difficult to derive the $z$ transform of the corresponding cardinal spline interpolator (expansion factor of two and shift by $\Delta$ )

$$
H(z)=\frac{B_{2, \Delta}^{n}(z)}{B_{1,0}^{n}\left(z^{2}\right)}
$$

where the sampled $B$-spline FIR kernels $B_{m, \Delta}^{n}(z)$ are defined as

$$
B_{m, \Delta}^{n}(z)=\sum_{k \in Z} \beta^{n}\left(\frac{k-\Delta}{m}\right) z^{-k} .
$$

The function $\beta^{n}(x)$ is the centered $B$-spline of degree $n$, which is constructed from the $(n+1)$-fold convolution of a rectangular pulse; an explicit formula is given by [2, Eq. (2.6)]. The $B$ splines are made up of polynomial segments of degree $n$ that are connected at the knot points; for $n$ odd, these knots are on the integers, whereas for $n$ even, they fall in between. The post filter (or synthesis filter) (2) specifies the EXPAND pyramid operator in the block diagram in Fig. 2(a). The coefficients of the least squares pyramid will be constructed by filtering and downsampling by a factor of two, as illustrated in Fig. 2(a) (REDUCE operation). The optimal analysis prefilter for the minimum error approximation in $S_{2}$ is

$$
\stackrel{\circ}{H}(z)=\frac{2 H\left(z^{-1}\right)}{H(z) H\left(z^{-1}\right)+H(-z) H\left(-z^{-1}\right)} .
$$

Proof: The least squares approximation of the signal $s_{1}(k) \in l_{2}$ is achieved when the error $s_{1}(k)-\tilde{s}_{1}(k)$ is perpendicular to $S_{2}=$ $\operatorname{span}\{h(k-2 l)\}_{l \in Z}$, or, equivalently, when

$$
\begin{aligned}
& \left\langle h(k-2 l), s_{1}(k)\right\rangle_{l_{2}} \\
& \quad=\left\langle h(k-2 l), \sum_{n \in Z} s_{2}(n) h(k-2 n)\right\rangle_{l_{2}}, \quad \forall l \in Z .
\end{aligned}
$$

We rewrite this identity using convolution and downsampling operators

$$
\left[h^{T} * s_{1}\right]_{\sqrt{ } 2}(k)=\left(a * s_{2}\right)(k)
$$

where $h^{T}(k)=h(-k)$, and $a(k)=\left[h^{T} * h\right]_{\downarrow 2}(k)$ is the autocorrelation sequence. Finally, we apply the inverse filtering operator $(a)^{-1}$, 


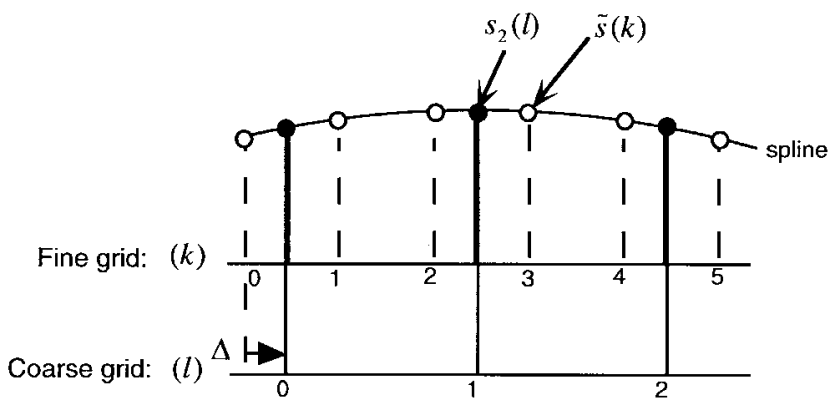

Fig. 1. Interpolation mechanism in the shifted spline pyramid. The lower resolution signal $\tilde{s} \in S_{2}$ is either represented by its coarse-grid samples $s_{2}(l)$ (black dots) or its fine-grid samples $\tilde{s}(k)$ (white dots) obtained by resampling the spline that interpolates $s_{2}(l)$. The underlying $B$-spline basis functions are centered on the coarse grid nodes. The mapping from $s_{2}(l)$ to $\tilde{s}(k)$ is implemented through the EXPAND function (upsampling + postfiltering).

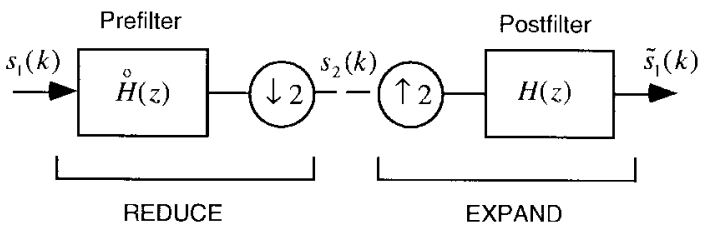

(a)

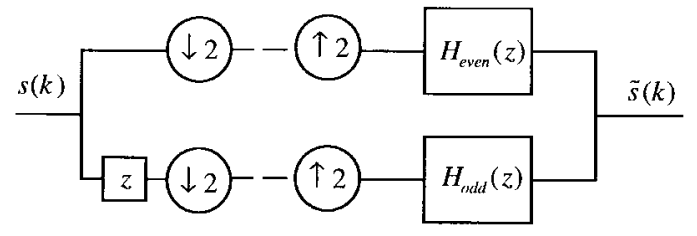

(b)

Fig. 2. Least squares pyramid. (a) Standard implementation of the REDUCE and EXPAND pyramid operations. (b) Equivalent polyphase representation of the approximation process.

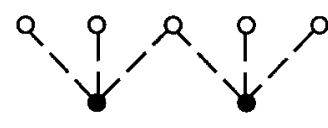

(a)
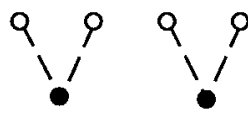

(b)
Fig. 3. Two primary examples of node configurations. (a) Symmetric pyramid $(\Delta=0)$. (b) Centered pyramid $(\Delta=1 / 2)$

which yields

$$
s_{2}(k)=(a)^{-1} *\left[h^{T} * s_{1}\right]_{\downarrow 2}(k)=\left[\stackrel{\circ}{h} * s_{1}\right]_{\downarrow 2}(k)
$$

where $\stackrel{\circ}{h}(k)=\left[(a)^{-1}\right]_{\uparrow 2} * h^{T}(k)$ is the filter whose $z$-transform is given by (4). The Riesz basis condition on $h(k-2 l)$ ensures that the inverse filter $(a)^{-1}$ is well defined.

There are two configurations of special interest (cf., Fig. 3): i) $\Delta=0$, in which case, all filters are symmetric (standard $l_{2}$ setup); and ii) $\Delta=1 / 2$, where the coarser level grid points are positioned at mid distance between two finer level nodes (centered pyramid). This latter quadtree-like topology may have advantages for multiscale edge detection or image segmentation because it facilitates the propagation of image labels from one level to the next. With our present convention, there is an equivalence between grid points and spline knots only when the degree $n$ is odd.

\section{Projection Operator AND PyRamid Characterization}

By definition, the global system in Fig. 2(a) (concatenation of the REDUCE and EXPAND operations) defines an orthogonal projector from $l_{2}$ into the sequence space $S_{2}$, which has half as many degrees of freedom. Since we are considering the two parameters $n$ and $\Delta$, this raises the issue of how to optimize the approximation space for the application at hand, for instance, image coding or multiscale signal processing. Energy compaction is a standard criterion that tends to suggest the use of higher order pyramids [11]. Here, we propose some additional tools and criteria for analyzing and comparing the various pyramids. These concepts are simple and universal and provide some further insights into the approximation process.

\section{A. Polyphase Analysis}

Let $s(k)$ and $\tilde{s}(k)$ denote the input and output of our system. Using the standard multirate signal processing identities, we write the system's equation in the $z$-transform domain

$$
\tilde{S}(z)=\frac{1}{2}(\stackrel{\circ}{H}(z) S(z)+\stackrel{\circ}{H}(-z) S(-z)) H(z) .
$$

Next, we replace the prefilter by its polyphase representation (cf. [12])

$$
\stackrel{\circ}{H}(z)=\stackrel{\circ}{H}_{0}\left(z^{2}\right)+z \stackrel{\circ}{H}_{1}\left(z^{2}\right)
$$

and manipulate (5) as

$$
\begin{aligned}
\tilde{S}(z)= & \frac{1}{2}\left(\stackrel{\circ}{H}_{0}\left(z^{2}\right) S(z)+z \stackrel{\circ}{H}_{1}\left(z^{2}\right) S(z)\right. \\
& \left.+\stackrel{\circ}{H}_{0}\left(z^{2}\right) S(-z)-z \stackrel{\circ}{H}_{1}\left(z^{2}\right) S(-z)\right) H(z) \\
= & \frac{1}{2}[S(z)+S(-z)] H_{\text {even }}(z)+\frac{z}{2}[S(z)-S(-z)] H_{\text {odd }}(z)
\end{aligned}
$$

where

$$
\begin{aligned}
H_{\text {even }}(z) & =\stackrel{\circ}{H}_{0}\left(z^{2}\right) H(z) \\
H_{\text {odd }}(z) & =\stackrel{\circ}{H}_{1}\left(z^{2}\right) H(z) .
\end{aligned}
$$

The block diagram interpretation of (7) is given in Fig. 2(b). The term $\frac{1}{2}[S(z)+S(-z)]$ (resp. $\frac{1}{2}[S(z)-S(-z)]$ ) represents the even (resp. odd) part of the signal where all samples with odd (resp. even) time indices have been set to zero. In this way, the system has been characterized by two transfer functions $H_{\text {even }}(z)$ and $H_{\text {odd }}(z)$, which act on the even and odd samples of the signal, respectively.

The even/odd transfer functions for the cases $n=3$ and $\Delta=0$ (symmetric cubic splines) (cf. [9]) and $n=3$ and $\Delta=1 / 2$ (centered cubic splines) are shown in Figs. 4(a) and (b), respectively. Interestingly, the moduli of the even and odd responses are identical for the centered case ( $n=3$ and $\Delta=1 / 2$ ), whereas they are not otherwise. The explanation for this phenomenon lies in the special symmetry of the underlying filters. Specifically, in the centered case $(\Delta=1 / 2)$, we have

$$
\stackrel{\circ}{h}(-k)=\stackrel{\circ}{h}(k-1) \leftrightarrow \stackrel{\circ}{H}\left(z^{-1}\right)=z^{-1} \stackrel{\circ}{H}(z)
$$

which implies that the even and odd polyphase components are reversed versions of each other, i.e., $\stackrel{\circ}{H}_{0}(z)=\stackrel{\circ}{H}_{1}\left(z^{-1}\right)$ (same modulus of their Fourier transform).

We have also observed that as the order of the spline increases, all filters converge to the ideal halfband filter with the appropriate phase shift. This is consistent with the property that splines converge to bandlimited functions as the degree goes to infinity [6], [13]. 


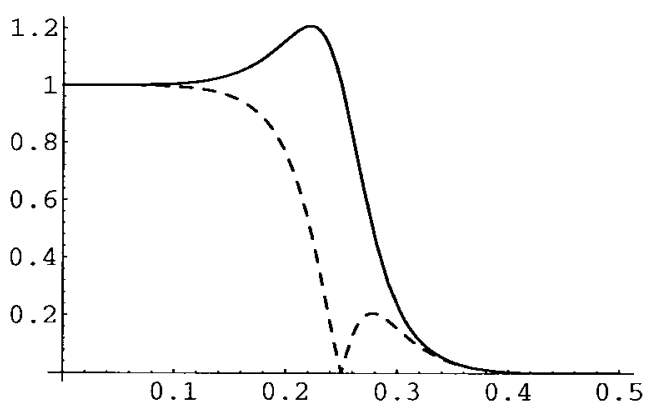

(a)

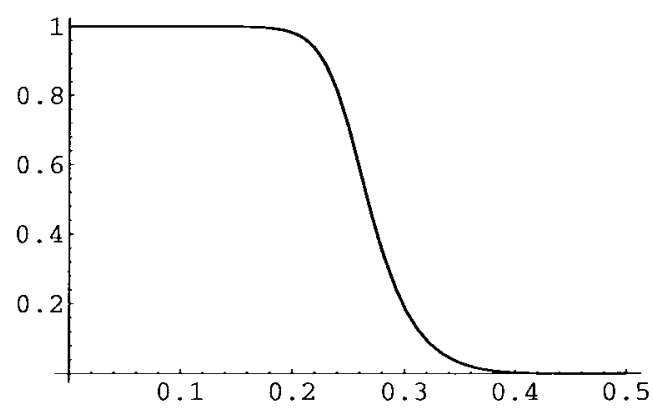

(b)

Fig. 4. Comparison of the even/odd frequency responses for the symmetric and centered cubic spline pyramids. (a) Even (solid) and odd (dashed) transfer functions for $n=3$ and $\Delta=0$. (b) Even and odd for $n=3$ and $\Delta=1 / 2$.

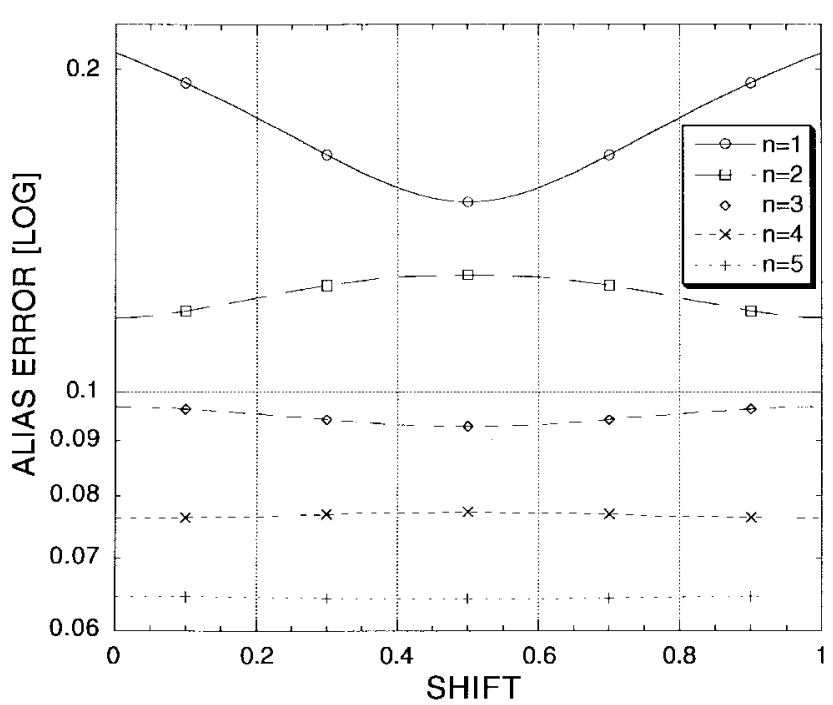

Fig. 5. Shift-invariance index $\alpha$ as a function of the shift $\Delta$ for splines of degree $n=1,2,3,4,5$.

\section{B. Shift Invariance}

In order to assess the degree of shift-invariance of the system, it is helpful to rewrite (5) as

$$
\tilde{S}(z)=\left(\frac{1}{2} \stackrel{\circ}{H}(z) H(z)\right) S(z)+\left(\frac{1}{2} \stackrel{\circ}{H}(-z) H(z)\right) S(-z) .
$$

The second term in (10) corresponds to the aliased part of the spectrum. Clearly, the system will be globally shift invariant if and only if the output can be written as $\tilde{S}(z)=G(z) S(z)$, that is, when the aliasing component is zero for any input $S(z)$. The degree of shift invariance will therefore depend on the magnitude of product $\frac{1}{2} \stackrel{\circ}{H}(-z) H(z)$, which should ideally be zero. Hence, we define the shift-invariance (or aliasing) index

$$
\alpha=\int_{0}^{1 / 2}\left|\stackrel{\circ}{H}\left(e^{j 2 \pi\left(f+\frac{1}{2}\right)}\right) H\left(e^{j 2 \pi f}\right)\right| d f .
$$

Note that this quantity is characterized in a way similar to the alias term in two-channel perfect reconstruction filterbanks (QMF) [3], [12]. In Fig. 5, we provide the graph of $\alpha$ as a function of $\Delta$ for splines of degree $n=1,2,3,4,5$. These plots were obtained by integrating (11) numerically for different values of $\Delta$. We observe that in the case of odd splines, the minimal value occurs for the shift $\Delta=1 / 2$ (centered pyramid), whereas in the case of even splines, it is $\Delta=0$ (symmetric pyramid). For a given $n$, the worst performance is achieved when the knots are positioned on the integers ( $\Delta=0$ for $n$ odd and $\Delta=1 / 2$ for $n$ even), which corresponds to the standard configuration where the spline spaces are nested. We conjecture that the same phenomenon should hold for higher order splines. Thus, it appears that the standard setup used in the context of the wavelet transform (Battle-Lemarié wavelets, $L_{2}$-spline pyramid) is suboptimal. From the graph, we also notice that the difference in performances decreases as we employ higher order splines. Again, this is not surprising since all splines representations asymptotically tend to the bandlimited model that is shift-invariant $(\alpha=0)$.

\section{CONCLUSION}

We have introduced spline pyramids using shifted multiresolution basis functions. The approximation is step-wise optimal in the least squares sense (minimum $l_{2}$-norm). Shifting one level of the pyramid with respect to the other may have advantages. In particular, we have shown that it can improve shift-invariance. We have also observed experimentally that a lower shift-invariance index is usually also associated with better energy compaction (the data is not shown here).

Interestingly, the standard spline configuration used in the context of the wavelet transform where the knots are positioned on the integers is the least favorable from the point of view of our performance criterion (shift-invariance). However, these differences vanish as the degree of the spline increases. In the limit as the order goes to infinity, the underlying projector is shift-invariant, irrespective of the shift parameter $\Delta(\alpha=0)$; it corresponds to a bandlimited signal approximation.

\section{REFERENCES}

[1] C. de Boor, A Practical Guide to Splines. New York: Springer-Verlag, 1978.

[2] M. Unser, A. Aldroubi, and M. Eden, " $B$-spline signal processing, Part I: Theory," IEEE Trans. Signal Processing, vol. 41, pp. 821-833, Feb. 1993.

[3] G. Strang and T. Nguyen, Wavelets and Filter Banks. Wellesley, MA: Wellesley-Cambridge, 1996.

[4] M. Unser, "Approximation power of biorthogonal wavelet expansions," IEEE Trans. Signal Processing, vol. 44, pp. 519-527, Mar. 1996.

[5] P. M. Prenter, Splines and Variational Methods. New York: Wiley, 1975.

[6] A. Aldroubi, M. Unser, and M. Eden, "Cardinal spline filters: Stability and convergence to the ideal sinc interpolator," Signal Process., vol. 28 , no. 2, pp. 127-138, Aug. 1992.

[7] S. G. Mallat, "A theory of multiresolution signal decomposition: The wavelet representation," IEEE Trans. Pattern Anal. Machine Intell., vol. PAMI-11, pp. 674-693, July 1989.

[8] M. Unser, A. Aldroubi, and M. Eden, "The $L_{2}$ polynomial spline pyramid," IEEE Trans. Pattern Anal. Machine Intell., vol. 15, pp. 364-379, Apr. 1993.

[9] M. Unser, A. Aldroubi, and M. Eden, " $B$-spline signal processing, Part II: Efficient design and applications," IEEE Trans. Signal Processing, vol. 41, pp. 834-848, Feb. 1993. 
[10] A. Aldroubi, M. Unser, and M. Eden, "Discrete spline filters for multiresolutions and wavelets of $l_{2}$," SIAM J. Math. Anal., vol. 25, no. 5, pp. 1412-1432, Sept. 1994.

[11] M. Unser, "On the optimality of ideal filters for pyramid and wavelet signal approximation," IEEE Trans. Signal Processing, vol. 41, pp. 3591-3596, Dec. 1993.

[12] M. Vetterli and J. Kovacevic, Wavelets and Subband Coding. Englewood Cliffs, NJ: NJ: Prentice-Hall, 1995.

[13] I. J. Schoenberg, "Notes on spline functions III: On the convergence of the interpolating cardinal splines as their degree tends to infinity," Israel J. Math., vol. 16, pp. 87-92, 1973.

\section{The Discrete Multiple Wavelet Transform and Thresholding Methods}

\author{
T. R. Downie and B. W. Silverman
}

Abstract-We propose thresholding for multiwavelets considering the coefficient vectors as a whole rather than thresholding individual elements. A multivariate universal threshold is obtained using the $\chi^{2}$ distribution. Simulations indicate that using the GHM multiwavelet with appropriate preprocessing, our method outperforms univariate thresholding of both GHM and Daubechies wavelet decompositions.

Index Terms-Multiwavlet, prefilter, smoothng methods, wavelet transforms, white noise.

\section{INTRODUCTION}

Multiple wavelets [8] have recently been formulated, using translations and dilations of $L \geq 2$ scaling functions $\phi_{1}, \cdots, \phi_{L}$ and $L$ mother wavelet functions $\psi_{1}, \cdots, \psi_{L}$. It has been proposed that multiwavelet bases should be better at wavelet applications than other wavelet bases [11].

We can approximate a function using a linear combination of the wavelet functions with vector coefficients $C_{j, k}=$ $\left(c_{j, k, 1}, \cdots, c_{j, k, L}\right)^{\top}$ and $D_{j, k}=\left(d_{j, k, 1}, \cdots, d_{j, k, L}\right)^{\top}$. Given the vector starting coefficients $C_{0, k}$, the wavelet coefficients can be found using the discrete multiple wavelet transform (DMWT) [13]. To obtain these starting coefficients, a method of mapping a sequence $f_{k}$ of univariate data to bivariate vectors has to be adopted.

A matrix prefilter does this by partitioning the data into a sequence of $L$ vectors and applying a filter defined by a sequence of $L \times L$ matrices $Q_{n}$. The starting coefficients are $C_{0, k}=$ $\sum_{n} Q_{n}\left(f_{L(n+k)}, \cdots, f_{L(n+k)+(L-1)}\right)^{\top}$. To recover the signal after reconstruction, we apply a postfilter, which is the inverse of the given prefilter. Therefore, if we apply a prefilter, DMWT, inverse DMWT, and postfilter to any sequence, the output will be identical to the input.

Alternatively, a repeated signal prefilter convolves a sequence of $L$ vectors $\gamma_{n}$ with the $f_{k}$. This is equivalent to sampling each

Manuscript received November 26, 1996; revised January 27, 1998. This work was mainly carried out at the University of Bristol while T. Downie was a Ph.D. student. It was completed while B. Silverman was a Fellow at the Center for Advanced Study in the Behavioral Sciences, Stanford University, Stanford, CA. This work was supported by the Engineering and Physical Sciences Research Council, U.K., and by NSF Grant SBR-9601236. The associate editor coordinating the review of this paper and approving it for publication was Dr. Truong Q. Nguyen.

T. R. Downie is with CMIS, CSIRO, North Ryde, Australia (e-mail: tim.downie@cmis.CSIRO.au).

B. W. Silverman is with the School of Mathematics, University of Bristol, Bristol, U.K. (e-mail: b.w.silverman@bristol.ac.uk).

Publisher Item Identifier S 1053-587X(98)05964-9.
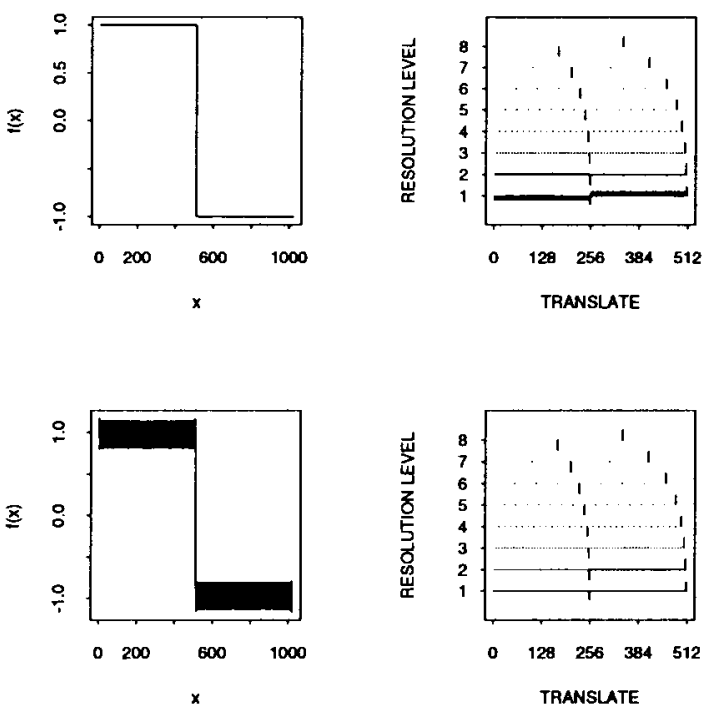

Fig. 1. Thresholding with the identity prefilter. (Top left) Piecewise constant signal. (Top right) Resulting wavelet coefficients using identity prefilter. (Bottom left) Reconstruction after thresholding these wavelet coefficients. (Bottom right) Wavelet coefficients using interpolation prefilter.

observation $L$ times using different weights. The starting coefficients are $C_{0, k}=\sum_{n} f_{n+k} \gamma_{n}$. When $L=2$, a repeated signal filter gives twice the number of coefficients as a matrix prefilter.

The identity prefilter assigns the data in blocks of length $L$ to the starting coefficients. Using a piecewise constant signal and the GHM multiwavelet [8], all the coefficients in level 1 have nonzero elements. Thresholding the small nonzero coefficients introduces a systematic high-frequency component after reconstruction (Fig. 1). A suitable prefilter overcomes this problem [7], [12], [13].

\section{Thresholding Multiple Wavelets}

Wavelet thresholding techniques [1], [3]-[5], [10] reduce the noise in an observed signal. Strela et al. [12] have applied the single wavelet (univariate) thresholding method [4] to a GHM multiwavelet decomposition with encouraging results. Even better results can be obtained by using a thresholding technique specifically for multiwavelets based on the multivariate properties of the decomposition.

Assume that the data $f_{i}$ are observations $g_{i}+\epsilon_{i}$ for $i=0, \cdots, N-$ $1\left(N=2{ }^{J} L\right)$, where $g_{i}$ is the signal observed at equally spaced discrete time points, and $\epsilon_{i}$ are independent $N\left(0, \sigma^{2}\right)$ noise. Although the DMWT is an orthogonal transformation, any prefilter except the identity prefilter will give correlated coefficients, and in particular, the elements within each coefficient may be highly correlated. As mentioned above, the identity prefilter gives poor results when thresholding. In addition, if there is a signal component present at a particular time-frequency location, then we would expect other elements in that coefficient to contain some signal component. Accordingly, the multivariate thresholding method accounts for the noise and signal components within the whole vector.

Applying the DMWT with an appropriate prefilter, we get $L$ vector coefficients $D_{j, k}=D_{j, k}^{*}+E_{j, k}$, where $D_{j, k}^{*}$ are the signal coefficients, and $E_{j, k}$ have multivariate normal distribution $N_{L}\left(0, V_{j}\right)$. The covariance matrix of the error term $V_{j}$ depends on the resolution level $j$. In the absence of any signal component, the quantity $\theta_{j, k}^{2}=D_{j, k}^{\top} V_{j}^{-1} D_{j, k}$ will have a $\chi_{L}^{2}$ distribution. Our 